\title{
Sexual Harassment and Gender Discrimination in Hospital Medicine: A Call to Action
}

\author{
Chithra R Perumalswami, MD, MSc ${ }^{*}$, Marisha Burden, MD²
}

${ }^{1}$ Center for Bioethics and Social Sciences in Medicine, University of Michigan Medical School, Ann Arbor, Michigan; ${ }^{2}$ Division of Hospital Medicine, Department of Medicine, University of Colorado School of Medicine, Aurora, Colorado.

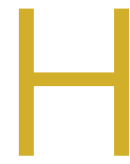

ospitalists are known as change agents for their fierce patient advocacy and expertise in hospital systems redesign. The field of hospital medicine has claimed numerous successes and the hospitalist model has been embraced by institutions across the country. Yet the lived experiences of hospitalists surveyed by Bhandari et al in this month's issue of JHM suggest a grim undertone. ${ }^{1}$ Hospital medicine is a field with high physician burnout rates, stark gender inequities in pay, leadership, and academic opportunities, and an unacceptably high prevalence of sexual harassment and gender discrimination. Women hospitalists disproportionately bear the brunt of these inequities. All hospitalists, however, can and should be an integral part of the path forward by recognizing the impact of these inequities on colleagues and hospital systems.

The study by Bhandari et al adds to the increasing body of knowledge documenting high levels of sexual harassment and gender discrimination in medicine and highlights important gender differences in these experiences among hospitalists nationally. ${ }^{1,2}$ Among 336 respondents across 18 academic institutions, sexual harassment and gender discrimination were both common and highly problematic within the field of hospital medicine, confirming what prior narratives have only anecdotally shared. Both men and women experienced harassment, from patients and colleagues alike, but women endured higher levels compared with men on all the measures studied.

Qualitative comments in this study are noteworthy, including one about a hospitalist's institution allowing potential faculty to be interviewed about plans for pregnancy, childcare, and personal household division of labor. One might argue that this knowledge is necessary for shift-based inpatient work in the context of a worldwide pandemic in which pregnant workers are likely at higher risk of increased morbidity and mortality. It remains illegal, however, to ask such questions, which are representative of the types of characteristics that constitute a toxic workplace environment. Moreover, such practices are particularly problematic given that pregnancy and childbearing for women in medicine come with their own set of well-documented unique challenges. ${ }^{3}$

The considerable body of research in this field should help guide new research priorities and targets for intervention. Does

*Corresponding author: Chithra R Perumalswami; Email: cperumal@umich. edu; Telephone: 734-615-7975; Twitter: @cperumalswami.

Received: October 21, 2020; Revised: November 1, 2020;

Accepted: November 1, 2020

๑ 2021 Society of Hospital Medicine DOI 10.12788/jhm.3561 the experience of sexual harassment impact hospitalists' intentions to leave their institutions or the career as a whole? Does sexual harassment originating from colleagues or from patients and families affect patient safety or quality of care? Do interventions in other international hospital settings specifically targeting respectfulness translate to American hospitals? ${ }^{4}$ These questions and a host of others merit our attention.

Hospital system leaders should work with hospital medicine leaders to support wholesale institutional cultural transformation. Implementation of antiharassment measures recommended in the 2018 report on sexual harassment from the National Academies of Sciences, Engineering, and Medicine is critical. ${ }^{2}$ This means supporting diverse, inclusive, and respectful environments at all levels within the organization, improving transparency and accountability for how incidents are handled, striving for strong and diverse leadership, providing meaningful support for targets of harassment, measuring prevalence over time, and encouraging professional societies to adopt similar actions. Furthermore, we believe it is critical to adopt a zero-tolerance policy for harassing behaviors and to hold individuals accountable. Encouraging all individuals within health care systems to uphold their ethical obligations to combat harassment and bias on a personal level is important. ${ }^{5}$ If left unaddressed, the unmet needs of those who are subjected to harassment and bias will continue to be problematic for generations to come, with detrimental effects throughout healthcare systems and the broader populations they serve.

Disclosures: The authors have nothing to disclose.

Funding: Dr Perumalswami is supported by grants from the $\mathrm{NIH}$, the Institute for Research on Women and Gender, and the University of Michigan Office of Research. Dr Burden is supported by grants from the AHRQ and the Gender Equity in Medicine Research Foundation.

\section{References}

1. Bhandari S, Jha P, Cooper C, Slawski B. Gender-based discrimination and sexual harassment among academic internal medicine hospitalists. J Hosp Med. 2021;16:XXX-XXX. https://doi.org/10.12788/jhm.3561

2. National Academies of Sciences, Engineering, and Medicine. Sexual harassment of women: climate, culture, and consequences in academic sciences, engineering, and medicine. National Academies Press; 2018. https://doi. org/10.17226/24994

3. Stentz NC, Griffith KA, Perkins E, Jones RD, Jagsi R. Fertility and childbearing among American female physicians. J Womens Health (Larchmt). 2016;25(10):1059-1065. https://doi.org/10.1089/jwh.2015.5638

4. Leiter MP, Laschinger HKS, Day A, Oore DG. The impact of civility interventions on employee social behavior, distress, and attitudes. J Appl Psychol. 2011;96(6):1258-1274. https://doi.org/10.1037/a0024442

5. Mello MM, Jagsi R. Standing up against gender bias and harassment - a matter of professional ethics. N Engl J Med. 2020;382(15):1385-1387. https:// doi.org/10.1056/nejmp1915351 